\title{
Action de divers Champignons entomophages sur Reticulitermes santonensis
}

Aperçu général

\author{
Par C. TOUMANOFF
}

Comme on le sait, la lutte contre les Insectes nuisibles revêt actuellement des aspects très variés, et la méthode biologique, qui comporte l'utilisation des insectes prédateurs ainsi que l'emploi des germes pathogènes, prend une ampleur de p'us en plus considérable.

L'utilisation de micro-organismes consiste à se servir des Bactéries, surtout des Bactéries aérobies sporogènes, et aussi des Champignons dits entomophages ou entomophytes qui détruisent les Insectes nuisibles, et qui, quoique pouvant se rencontrer à l'état libre (dans le sol, les matières végétales en décomposition, etc...), manifestent une prédilection pour le corps des divers Insectes.

On peut distinguer ainsi deux types de la lutte microbiologique, contre les Insectes nuisibles : la lutte bactériologique et la lutte fongique.

La lutte bactériologique a fait pendant ces dernières années des progrès considérables.

Ce sont surtout les Bactéries aérobies sporogènes appartenant à l'espèce Bacillus cereus (var. alesti, thuringiensis, etc...) qui ont été utilisées avec succès contre les chenilles des Lépidoptères.

Cette application a été précédée de recherches en laboratoire, qui ont permis de définir le mécanisme de l'effet pathogène et les caractéristiques des diverses souches bactériennes actuellement en usage.

Il fut démontré que l'action pathogène exercée par certaines Bactéries atteste généralement une spécificité, et que les Bactéries, très pathogènes pour les Lépidoptères par exemple, sont le plus souvent, à quelques exceptions près, sans effet sur les Insectes appartenant à d'autres groupes.

La spécificité d'action des Bactéries est bien plus manifeste que celle des Champignons, ces derniers s'attaquant parfois à des hôtes aussi différents que les larves des Lépidoptères, les larves et les adultes de certains Coléoptères, Hémiptères, Orthoptères. 
Cette circonstance fait des Champignons un matériel de choix pour la lutte contre divers Insectes ravageurs des plantes et du bois, et, comme nous le verrons, contre les Termites, qui nous intéressent tout particulièrement.

Comme on le sait, la vie des Insectes est souvent étroitement associée à l'existence des Champignons, et « vice-versa ».

En effet, si les Champignons se rencontrent parfois en association avec les Insectes, cela ne signifie pas qu'ils sont parasites.

Entre le parasitisme vrai, qui entraîne la mortalité importante et plus ou moins rapide des Insectes, et la symbiose, il existe certainement une action parasitaire atténuée.

Les Champignons, tels que les espèces des genres Cladosporium, Fusarium, etc..., ne nuisent le plus souvent que peu aux Insectes, mais peuvent contribuer à leur mortalité.

Les Champignons parasites vrais des Insectes, ou Champignons entomophytes ou entomophages, appartiennent à trois grandes subdivisions des Oomycètes, des Ascomycètes et aussi des Hyphomycètes.

Ce dernier groupe comporte les Champignons qui se reproduisent par les conidies. Parmi les Oomycètes, il faut signaler les Entomophtorées, qui sont des parasites importants d'Insectes.

Il en est de même en ce qui concerne les Hyphomycètes.

Dans plusieurs travaux antérieurs, nous avons eu l'occasion de traiter de l'action des Champignons sur les Insectes.

En ce qui concerne les Termites de France, aucune étude systématique n'a été faite quant à la possibilité de leur infection par les Champignons.

Chaz le Termite de Saintonge (Reticulitermes santonensis de Feytaud), on n'a observé qu'une seule fois l'infection mycosique, et il s'agissait, en l'occurrence, d'une espèce nouvelle.

En effet, Roger Heim et Harro Buchli ont pu isoler des Termites un Champignon qui est spécifique à ces Insectes. Il s'agit en l'occurrence d'un champignon ressemblant ̀̀ un Hyphomycète, Hormiscium, mais dont la morphologie diffère de celle des représentants de ce genre, et que ces auteurs ont dénommé Antennopsis gallica Heim et Buchli, qui est le type du nouveau genre Antennopsis. Par la suite, après une étude plus approfondie de cette forme et sa comparaison avec les représentants des divers ordres des Champignons, Heim conclut que ce Champignon ne saurait être rattaché aux Hyphomycètes et propose la création d'un ordre nouveau, celui de Gloehaustoriales, dans lequel on doit incorporer le genre Antennopsis. On trouvera dans la publication de Heim et Buchli la description de l'espèce Antennopsis gallica, ainsi que la justification de la création de l'ordre nouveau de Gloehaustrales.

Nous rappellerons ici seulement les constatations de Heim et Buchli concernant l'effet de ce Champignon sur les Termites. Cet effet est ainsi résumé par Heim : «Triple effet du parasite sur l'Insecte qu'il affaiblit et dont il ralentit les mouvements, dont il rend la mue difficile, enfin qu'il gêne dans ses déplacements par suite de la sécrétion, par les cellules fongiques, d'une substance muqueuse qui forme un enduit visqueux au contact duquel les divers organes des Termites se collent l'un à l'autre. » 
Dans le cas de l'infection par ce Champignon, les Termites appartenant aux différentes castes portaient « des conidiophores dressés, brun foncé, parfois abondants, poussant sur les diverses parties du corps». Toutefois, ils notent aussi que: "L'examen anatomique d'Insectes gravement atteints ne révèle la présence d'aucun hyphe ni d'altération histologique. »

Cette forme d'infection est très spéciale et différente de celles provoquées par les Champignons entomophages connus. Il s'agit là, en effet, d'un processus parasitaire consistant en la sécrétion de mucus qui eng!obe le corps de l'Insecte et l'immobilise, sans aucune forme de lésion des tissus ou de réactions tissulaires, à la suite de l'action du parasite. D'après Heim et Buchli, l'application de ce Champignon se heurterait actuellement à un certain nombre de difficultés et notamment la possibilité de la préparation des cultures en masse de ce Champignon, ainsi que son introduction directe dans les nids à Termites qui nécessiterait de p.us amples connaissances sur son cycle.

Nous avons isolé des Termites morts les Champignons des genres Mucor et Fusarium, dont les noms spécifiques n'ont pas été reconnus. Le Mucor s'est montré sans action sur les Termites, mais Fusarium s'est montré faiblement pathogène pour ces Insectes.

Aucun Champignon, parmi ceux qui sont qualifiés comme des entomophages vrais, n’a été à notre connaissance mis jusqu'ici en évidence chez la forme du Termite de Saintonge.

Cette forme de Termite, Reticulitermes lucifigus, var. santonensis, particulièrement nuisible par l'invasion et la destruction des maisons, a été très bien étudiée par de Feytaud.

Nous nous dispenserons ainsi de donner un aperçu sur la biologie de cet Insecte, bien traitée par de Feytaud et d'autres auteurs, non plus que les indications sur l'attaque des habitations par ces Insectes et sa propagation ; celles-ci sont également exposées dans diverses publications; nous ne citerons que les faits qui nous semblent intéressants et en rapport avec notre étude.

L'un des traits principaux du comportement de cet Insecte, qui nous intéresse tout particulièrement (sur le plan de son infection), est que l'individu affaibli, ou mort, d'une colonie, est rapidement déchiqueté et dévoré par ses congénères.

Cette circonstance doit favoriser l'extension d'une affection, et, en même temps, rend difficile la recherche dans la colonie des Insectes morts ou malades. C'est ainsi que, dans la nature, il est difficile de trouver les cadavres, et ce n'est qu'exceptionnellement qu'on les rencontre. Par contre, on constate que les Termites de Saintonge abandonnent fréquemment les lieux de leur développement, et notamment les galeries qu'ils construisent le long des troncs d'arbres, où normalement on observe un cheminement très actif d'Insectes.

Il en est de même en ce qui concerne d'autres lieux de développement : les pièges en carton ou en bois, les copeaux et les boiseries.

Afin de reconnaître l'effet des Champignons sur les Termites de Saintonge, nous avons utilisé des souches qui ont été obtenues des laboratoires d'Europe occidentale et orientale (Hollande, Tchécoslovaquie, U.R.S.S., etc...). 
Nous avons utilisé dans nos expériences surtout les Champignons entomophages vrais, isolés d'Insectes, appartenant au groupe d'Hyphomycètes, une seule espèce d'Oomycètes (Entomophtora sp. reçue de Tchécoslovaquie), un Phycomycètes (ordre des Mucorales) genre Mucor (Mucor sp.) et un représentant du genre Fusarium, les deux derniers isolés par nous-même des cadavres de Termites morts apparemment d'une infection.

\section{Techniques d'Essais et Résultats}

Nous avons utilisé pour nos expériences les Champignons infectants cultivés sur le milieu Sabouraud liquide et solide et aussi sur le milieu d'épreuves de l'effet antibiotique des Champignons.

Il est inutile de rapporter ici la composition du milieu de Sabouraud ; nous rappellerons toutefois celle du dernier milieu, «celui de Screening», dont voici la composition : glucose pur $10 \mathrm{~g}$, peptone pancréatique $5 \mathrm{~g}$, CINa $5 \mathrm{~g}$, eau déminéralisée q.s.p. $1.000 \mathrm{cc}, \mathrm{pH}$ généralement 7,5 , parfois varié.

L'expérimentation a été conduite avec les cultures simples ou les cultures liquides agitées pendant 72 ou 96 heures.

Les expériences effectuées ont attesté plusieurs modalités dont voici les principales:

$1^{\circ}$ la mise en contact des Termites avec les cultures de Champignons dans des boîtes de Pétri, où ils demeuraient jusqu'à leur mort ;

$2^{\circ}$ les Termites ont été contaminés par la sciure humectée de culture du Champignon.

Les résultats des expériences effectuées sur des centaines d'Insectes sont résumés ci-dessous.

Nous rapporterons tout d'abord les expériences de mise en contact des Termites avec les cultures de Champignons, cultivés dans des boîtes de Pétri en milieu solide.

\section{Metarrhizium anisopliae (METCH) SOROKIN.}

Avec ce Champignon (originaire de Tchécoslovaquie) la mortalité des Termites a commencé au laboratoire en quarante-huit heures et fut totale en quatre jours. Les symptômes de l'infection, qui ne se manifestent qu'au bout de quarante-huit heures, ont consisté en un ralentissement de l'activité de l'Insecte, parésie, puis l'immobilisation qui fait penser à la paralysie, qui ne survient que lentement.

Une souche d'une autre origine a donné des résultats beaucoup plus manifestes, la mortalité commençait déjà vingt-quatre heures après le contact et fut totale en quarantehuit heures.

Dans certaines expériences, cependant, pratiquées avec la souche de Tchécoslovaquie, la mortalité a été observée également au bout de vingt-quatre heures.

Quant aux symptômes, ils étaient semblables avec les souches différentes de cette espèce. 


\section{Beauveria bassiana (BALSAMO) VIEULLEMIN.}

Les Termites mis en contact avec la culture BB (culture de 34 jours), déjà, quarante-huit heures après le contact, manifestent les symptômes suivants : convulsions, mouvements désordonnés, suivis de la mort.

Nous avons constaté, dans un cas, un début de la mortalité au bout de dix-huit heures, et la mortalité de tous les Insectes en vingt-quatre heures.

Toutefois, les expériences répétées démontrent qu'il n'en est pas toujours ainsi dans les mêmes conditions.

En effet, les Termites n'éprouvent parfois aucune gêne après un contact avec le Champignon durant vingt-quatre heures, et la mortalité ne débute qu'au début du troisième jour, après le contact avec le Champignon, pour s'achever le cinquième jour.

Il arrive parfois que la mortalité se produit dans un délai très court, au bout de trois heures, après avoir mis les Termites en contact avec la culture de Beauveria bassiana, alors que la mortalité totale se produit dans le délai de vingt-quatre heures.

\section{Beauveria globulifera (PICARD) 1913.}

Ce Champignon, qui s'apparente au précédent, a été utilisé en culture d'un mois, sur le milieu Screening.

Les expériences réalisées démontrèrent qu'une certaine variabilité s'observe individuellement en ce qui concerne les modalités d'infection des Termites par ce Champignon.

C'est ainsi qu'au cours d'une expérience, la mortalité des Insectes a commencé à se manifester au bout de dix-huit heures, pour atteindre le point culminant, c'est-à-dire leur destruction totale, au bout de vingt-quatre heures.

Dans un autre essai avec une culture de même âge, le début de la mortalité, qui s'est élevée à $50 \%$, a commencé au bout de vingt-quatre heures et la mortalité totale survint quarante-huit heures après le début des expériences.

Enfin, dans le troisième essai, la mortalité a commencé à se manifester au bout de vingt-quatre heures, et atteint $60 \%$. Les $40 \%$ de Termites restants moururent quarante-huit heures plus tard, c'est-à-dire soixante-douze heures après le point de départ des expériences.

Dans le cas de l'infection des Termites par ce Champignon les symptômes n'ont pas été les mêmes que ceux provoqués par l'utilisation des Champignons précédents.

En effet, nous avons assisté en l'occurrence à des comportements différents :

$1^{\circ}$ la mortalité sans convulsions préalables;

$2^{\circ}$ la mort précédée de convulsions légères (et il faut remarquer que les vieilles cultures provoquent invariablement des convulsions, contrairement aux jeunes cultures).

La dénomination des champignons est faite d'après la nomenclature utilisée par le Centraalbureau voor Schimmelcultures (Hollande) d'où provenaient la plupart des souches éprouvées sur les termites.

Lorsqu'une espèce était d'une autre origine, cela est mentionné dans le texte. 
Beauveria tenella (DELACR) SIEM.

Ce Champignon, qui appartient au même genre que les deux précédents, est moins pathogène pour les Reticulitermes (mise en contact avec les cultures).

En effet, aucune mortalité n'a été enregistrée avant le cinquième jour, et les Insectes mouraient, en général, le sixième jour après le début des expériences.

\section{Paecilomyces javanicus (FrIED. et BALLY) Brown et SMITH.}

Dans ce cas on utilise une culture (gélose Screening) âgée environ d'un mois ; la mort des Insectes est très rapide, c'est-à-dire vingt-quatre heures après la mise en contact de la culture et au plus tard quarante-huit heures après.

\section{Paecilomyces elegans (Cda) MAsON et Hughes.}

Cette espèce, utilisée en culture de même âge que la première, provoque une mortalité tardive, et les Insectes vivent en contact permanent avec le mycélium ; la mortalité, quoique partielle, ne se manifeste qu'au bout de quatre-cinq jours dans certaines expériences, et s'achève en général le dixième jour.

\section{Paecilomyces marquandï (MASSEE) HUGHES.}

La culture de ce Champignon a donné des résultats variables et la mortalité se manifeste tantôt au bout de vingt-quatre heures, tantôt plus tard, et s'achève en huit jours.

\section{Paecilomyces carneus (Duche et HeIm) Brown et G. SMITH.}

Certains Insectes placés en contact avec la culture étaient enrobés de celui-ci.

Cette espèce de $P$. provoque la mort en 48-72 heures et tous les insectes meurent en général en quatre-cinq jours.

\section{Paecilomyces fumoso-roseus (WIZE) BRown et G. SMITH.}

Cette dernière espèce de Champignon a montré le même effet pathogène que la précédente, la mort survenait de quarante-huit à cinq jours.

Les Termites semblaient, en l'occurrence, éviter le mycélium, et creuser des galeries dans la gélose environnante.

\section{Spicaria prasina (MAUBL.) SAW.}

Cette espèce pathogène pour d'autres Insectes (culture gélose Screening) s'est avérée également toxique par contact avec le Reticulitermes.

La mortalité commença quarante-huit heures après le contact des Insectes avec le mycélium du Champignon et la mortalité fut totale en soixante-douze heures. 


\section{Spicaria rubido-purpurea AОКI.}

Avec cette espèce, la mortalité commença à se manifester à partir du quatrième jour et s'est échelonnée sur les dix jours.

\section{Cephalosporium (sp.)*.}

Ce Champignon (culture sur gélose Sabouraud) exerce un effet plutôt lent sur les Termites avec lesquels il est mis en présence. Ces Termites commencent à mourir au bout de trois jours; leur mortalité atteint $50 \%$ au bout de dix jours seulement, et fut totale au bout de seize jours.

\section{Myrothecium verrucaria (ALB. et Schw.) DITMAR et Fr.}

Cette espèce, peu connue en tant qu'entomophage, provoque expérimentalement la mortalité du Termite après des délais d'exposition variant de vingt-quatre heures à six jours. Dans ce cas, les Termites creusent des galeries et meurent sans manifester de symptômes saisissables.

\section{Fusarium (sp.).}

Ce Champignon, qui est considéré généralement comme étant un « semi-parasite » de par la tolérance que manifestent à son égard les Insectes, provoque la mortalité de quelques Termites au bout de trois jours, mais, dans l'ensemble, les Insectes se montrent résistants à l'égard de ce germe et vivent quinze jours et même davantage. Ils ne meurent que lorsque le Champignon, qui pousse généralement sous la forme d'un enduit visqueux, finit par les englober en empêchant leur respiration. La mort, en l'occurrence, pourrait être attribuée plutôt à la souillure des téguments qu'à une infection réelle.

Une série d'autres essais a été faite avec les cultures de divers Champignons, en milieu liquide et aéré par agitation. Il serait fastidieux et inutile de rapporter ces essais qui ont donné sensiblement les mêmes résultats.

Les résultats ont été manifestement les mêmes avec les souches cultivées sur milieux solides et liquides. Notons qu'il était établi que l'addition à la culture du Champignon cultivé en milieu liquide agité, de $2 \%$ de cellulose, favorise l'infection, qui se produit toutefois aussi bien par la mise des Termites dans la sciure additionnée d'une culture liquide du Champignon.

D'autre part, une série d'expériences sur l'infection des Termites a été faite en utilisant des Champignons ensemencés avec des Bactéries. Plus précisément, il s'agissait de culture dans des boîtes de Pétri, où le Champignon fut ensemencé sous forme d'un trait d'une manière appropriée pour le «Screening », et, perpendiculairement à cette strie, ont été ensemencés les germes suivants : Salmonella, Shigella, Staphylocoque, et aussi deux souches de Vibrion cholérique. Les cultures ainsi réalisées, vieilles de six

- Tchécoslovaquie, Prague: Simensakova. 
mois ou davantage, dont la gélose « Screening » était déjà fortement desséchée, se sont avérées extrêmement toxiques pour les Termites.

En effet, les Insectes déposés dans les boîtes de Pétri commençaient à manifester une agitation anormale au bout d'une heure ou deux, puis une très forte agitation et du « tremor » comparable à celui que les insectes présentent en cas d'action des insecticides organiques de contact. Cette association des Bactéries avec un Champignon a produit une action particulièrement efficace et rapide avec Metarrhizium anisopliae et aussi Beauveria bassiana.

Nous relaterons plus tard ce curieux effet des associations Champignon + Bactéries sur les Termites, que nous nous contentons pour le moment de signaler. Il est possible que la substance antibiotique secrétée par le Champignon à l'égard d'un ou plusieurs des germes utilisés soit toxique pour les Insectes.

La lyse bactérienne, qui libère la ou les toxines qui se diffusent dans le milieu, est fatale pour les Insectes.

Notons enfin que divers extraits de Champignons, employés pour la détection de leur effet antibiotique antibactérien, ont été utilisés contre les Termites.

\section{Conclusions}

Nos très nombreuses expériences nous autorisent à conclure que :

$1^{\circ}$ La plupart des Champignons entomophages aue nous avons utilisés, sont, dans les conditions de laboratoire, extrêmement pathogènes pour Reticulitermes santonensis et provoquent des mortalités chez cet Insecte, mortalités qui s'échelonnent, selon les cas, entre quelques heures et quelques jours.

$2^{\circ}$ L'infection des Termites, à juger par les études histologiques $\left(^{*}\right)$, atteste deux modalités :

1) le simple contact aboutissant à la mort ;

2) la pénétration des hyphes dans la cavité générale.

$3^{\circ}$ Les Insectes morts se recouvrent d'un revêtement plus ou moins caractéristique selon l'espèce de Champignon utilisé.

Le premier mode d'infection mortelle n'a jamais été constaté jusqu'à présent au cours d'études sur l'infection expérimentale des Insectes par le Champignon. On peut concevoir en l'occurrence deux modes d'action :

1) l'effet toxique direct des spores du Champignon mises en contact avec le tégument de l'Insecte ;

2) la germination des spores en contact avec le tégument, et libération de la substance toxique se diffusant à travers le tégument, et tuant l'Insecte assez rapidement.

- Un certain nombre de coupes histologiques ont été effectuées au Service de M. Levaditi, à l'Institut Pasteur, à qui nous adressons nos remerciements. 
Le second mode d'affection est comparable à celui que l'on observe dans les cas d'infection d'autres Insectes, et notamment des Lépidoptères par les Champignons entomophages.

Dans les deux cas la mort survient avant l'envahissement total du corps de l'Insecte.

En ce qui concerne Reticulitermes santonensis, le corps de l'Insecte est envahi «post-mortem » par les hyphes des Champignons, et recouvert d'un enduit poudreux constitué par des spores.

Ce revêtement atteste un aspect caractéristique dans la plupart des cas pour chaque souche infectante.

On observe, par conséquent, la «momification» des Termites par les Champignons à la suite de l'infection expérimentale ; la momification peut certainement se produire aussi dans la nature.

Ce revêtement aboutit quelquefois à l'expansion du mycélium dans le milieu où sont placés les Termites (sciure humectée de culture), ce qui doit contribuer à la dissémination du Champignon et à sa propagation.

$4^{\circ}$ Dans l'ensemble, la plupart des Champignons entomophages vrais sont pathogènes pour Reticulitermes santonensis; toutefois il est à noter que ce sont les Hyphomycètes : Metarrhizium anisopliae (Metch) Sorokin et Beauveria bassiana (Balsamo) Vieullemin qui ont l'action la plus manifeste et la plus persistante.

Ainsi ce sont surtout ces deux espèces qui, s'attaquant entre autres aux Insectes habitant dans le sol, ont été retenues pour des expériences ultérieures et des essais sur le terrain.

\section{Bibliographie}

\section{Biologie.}

1) Feytaud DE, 1912. - Contribution à l'étude du Termite lucifuge. Thèses présentées à la Fac. des Sc. de Paris, Masson et $\mathrm{C}^{\text {ie }}$, Edit.

2) —, 1957. - Una malattia degli edifici : La Termitosi (Trad. Prof.; G. Bonaventure), Roma.

3) —, 1921. — La cité des Termites, Paris, Librairie L. Homme.

4) —,1921. - La cité des Termites. Mours sociales du Termite lucifuge, ses ravages, sa destruction. Paris, Librairie L. Homme. Bordeaux, Librairie Féret et fils.

5) - 1959. - A propos de la menace des Reticulitermes, Revue du bois, Strasbourg.

6) —, 1959. - L'histoire véridique du Termite de Saintonge. Cahier de l'Ouest, $\mathrm{n}^{\circ}$ 30, juillet.

\section{Pathologie.}

Heim (R.) et BuCHLI H., 1951. - Un champignon parasite du Termite de Saintonge. C.R. Acad. Sc., séance 22-1-51, T. 232, pp. 277-280. 
Heim (R.), 1951. - Le nouvel ordre des Gloeohaustoriales. C.R. Acad. Sc., séance 19-111951, T. 233, pp. $1245-1248$.

Madelin M. P., 1963. - Diseases caused by Hyphomycetous Fungi in «Insect Pathology ». An advanced Treatese, vol. II, edited by E. Steinhaus. Acad. Press, New York-London. (On trouvera dans cette publication une bibliographie très complète sur la question).

Martinez J. B., 1958. - Los termes en España. Servicio de Plagas Forestales, Madrid.

PICARD F., 1914. - Les Champignons parasites des Insectes et leur utilisation agricole, Coulet et Fils, édit., Librairie de l'Ecole Nationale d'Agriculture, Montpellier.

ToumanofF C., 1928. - On the infection of Pyrausta nubilalis Hubn. by Aspergillus flavus L. and Spicaria farinosa. Inter. Corn Borer Invest. Scientific Reports.

-, 1933. - Action des Champignons entomophytes sur la pyrale de maïs (Pyrausta nubilalis Hubn.). Ann. de Parasit. Hum. et Compar., t. XI, $\mathrm{n}^{\circ}$ 2, mars, pp. 6, microph. 

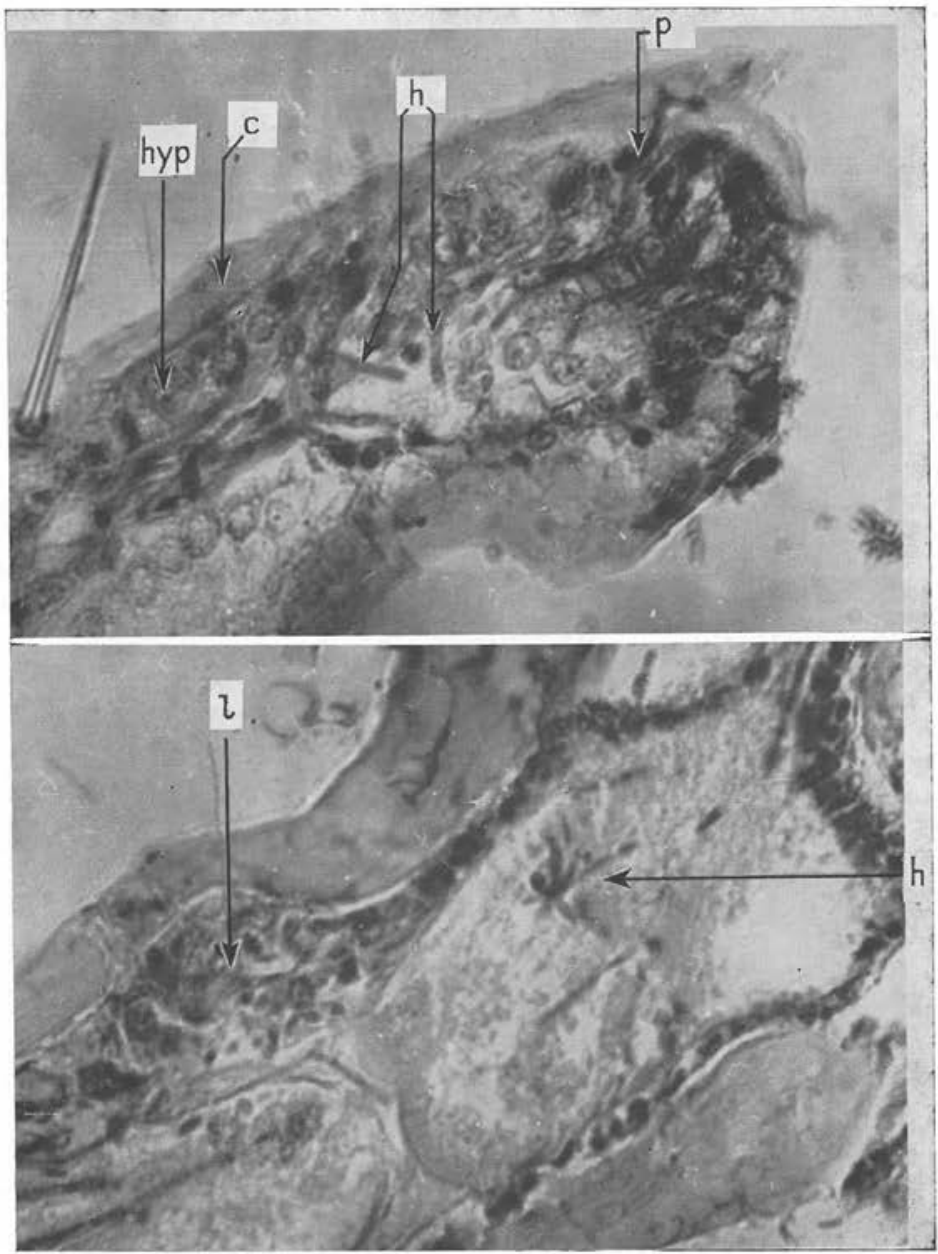

PL. I. - Photomicrographies montrant l'aspect d'infection des Termites par le Champignon entomophage. Il s'agit du procédé commun pour la plupart des espèces utilisées. En haut : $c$..., revêtement chitineux du tégument, montrant le passage du Champignon à travers la cuticule chitineuse dans l'hypoderme et la cavité générale (p.; h. et hyp., hyphes, c., cuticule). En bas : sensiblement la même image avec un amas de leucocytes dégénérés à l'endroit de la pénétration du Champignon (l.) et hyphes de la cavité générale (h.). 

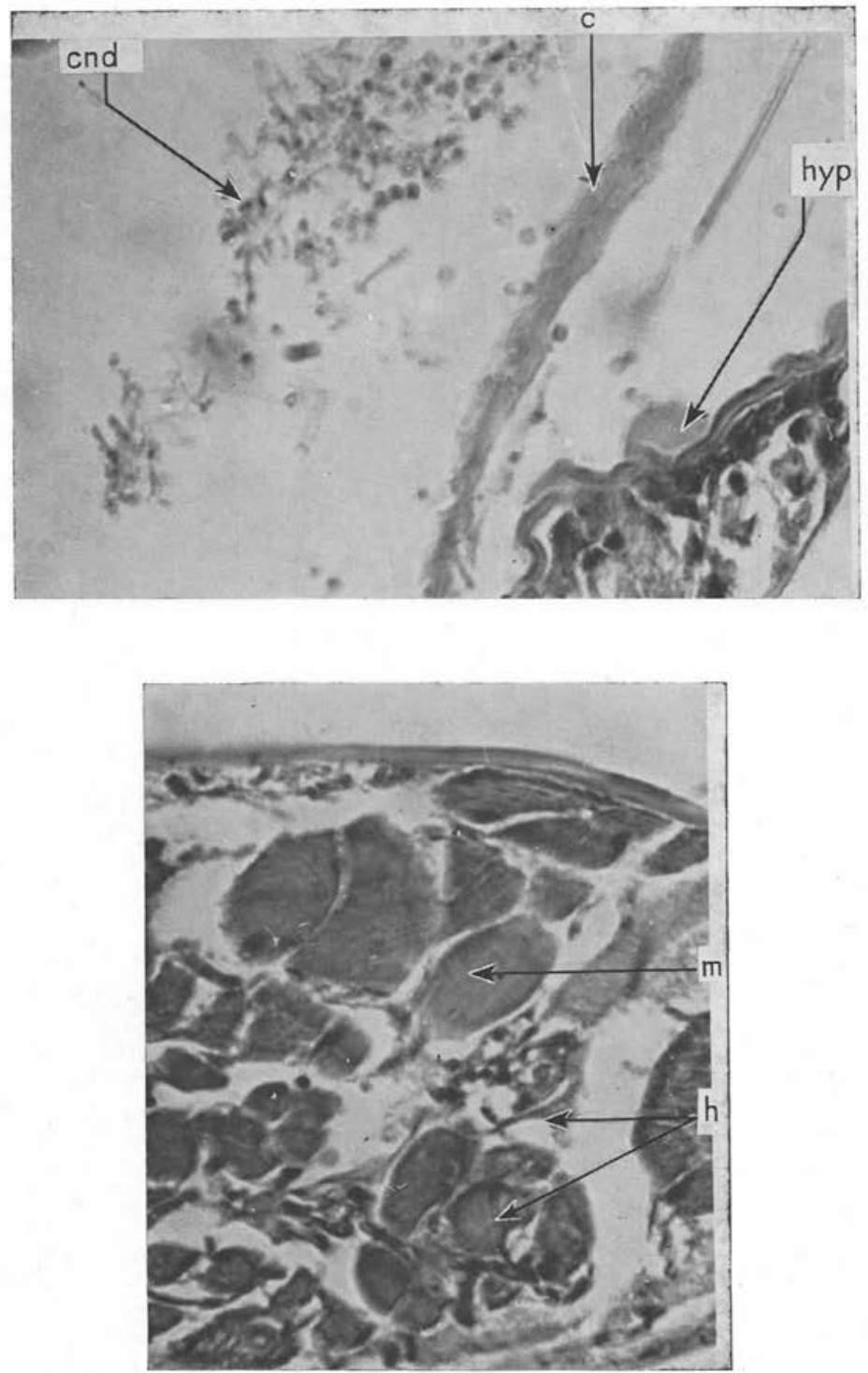

PL. II. - En haut, une coupe montrant: la cuticule chitineuse détachée de l'épithélium chez un Termite mourant et à gauche l'amas de conidies, en grande partie en voie de germination. La mort de l'Insecte est survenue sans pénétration du Champignon dans la cavité générale du corps, par simple contact des conidies avec le tégument. En bas: coupe passant dans une extrémité de Termite; on voit les hyphes parmi les tronçons des muscles en partie disloqués. (cnd., conidies ; c., cuticule; h. et hyp., hyphes ; m., muscles). 

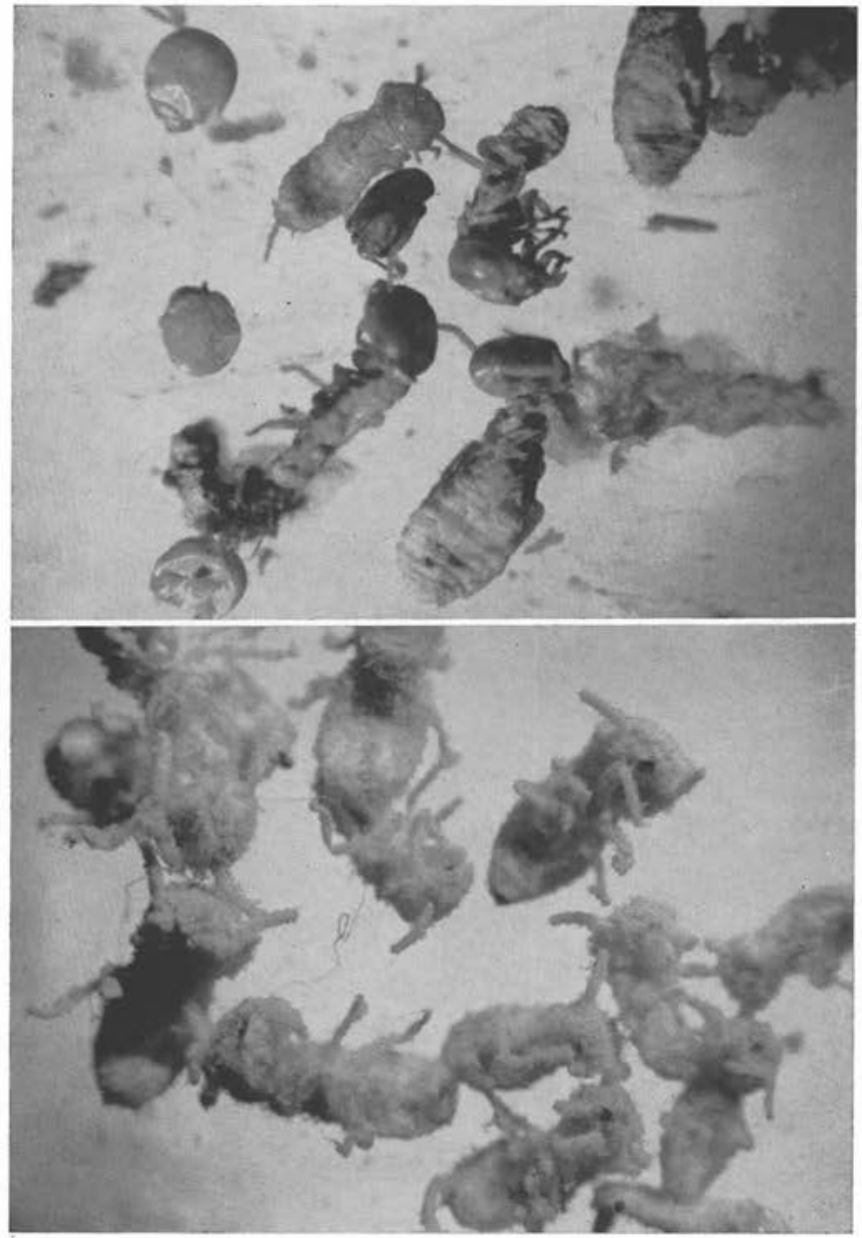

PL. III. - Aspect des cadavres de Termites infectés par : en haut, Beauveria tenella; en bas, Beauveria globulifera 

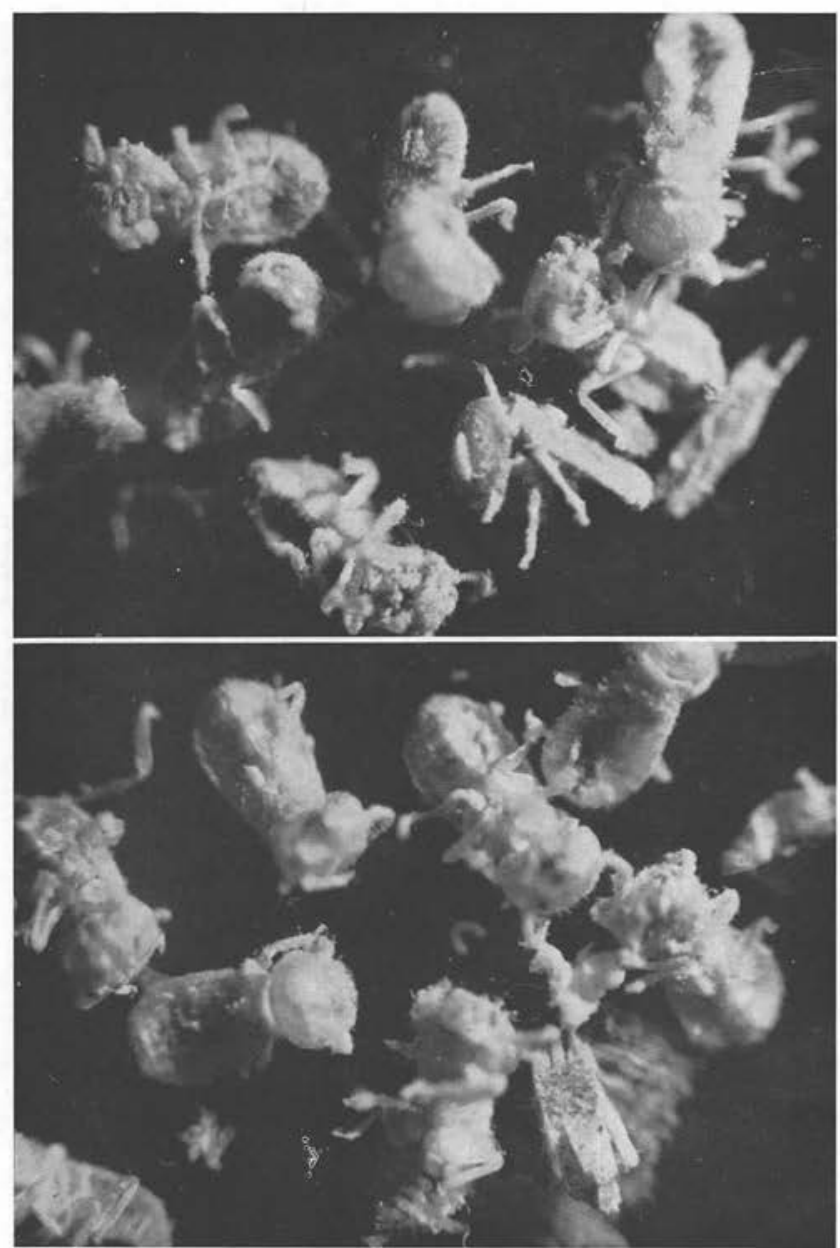

PL. IV. - Aspect des cadavres de Termites infectés par: Metarrhizium anisopliae ; 14) Beauveria bassiana 